\title{
Prothrombin complex concentrates and a specific antidote to dabigatran are effective ex-vivo in reversing the effects of dabigatran in an anticoagulation/liver trauma experimental model
}

\author{
Oliver Grottke ${ }^{1 *}$, Joanne van Ryn², Henri MH Spronk ${ }^{3}$ and Rolf Rossaint ${ }^{1}$
}

\begin{abstract}
Introduction: New oral anticoagulants are effective alternatives to warfarin. However, no specific reversal agents are available for life-threatening bleeding or emergency surgery. Using a porcine model of trauma, this study assessed the ability of prothrombin complex concentrate (PCC), activated PCC (aPCC), recombinant FVIla (rFVIla) and a specific antidote to dabigatran (aDabi-Fab) to reverse the anticoagulant effects of dabigatran.

Methods: Dabigatran etexilate (DE) was given orally for 3 days $(30 \mathrm{mg} / \mathrm{kg}$ bid) and intravenously on day 4 to achieve consistent, supratherapeutic concentrations of dabigatran. Blood samples were collected at baseline, after oral DE, after intravenous dabigatran, and 60 minutes post-injury. PCC (30 and $60 \mathrm{U} / \mathrm{kg}$ ), aPCC (30 and $60 \mathrm{U} / \mathrm{kg}$ ), rFVIla (90 and $180 \mathrm{\mu g} / \mathrm{kg}$ ) and antidote (60 and $120 \mathrm{mg} / \mathrm{kg}$ ) were added to blood samples ex-vivo. Coagulation was assessed by thromboelastometry, global coagulation assays and diluted thrombin time.
\end{abstract}

Results: Plasma concentrations of dabigatran were $380 \pm 106 \mathrm{ng} / \mathrm{ml}$ and $1423 \pm 432 \mathrm{ng} / \mathrm{ml}$ after oral and intravenous administration, respectively, and all coagulation parameters were affected by dabigatran. Both PCCs and aDabi-Fab, but not rFVIla, reversed the effects of dabigatran on thromboelastometry parameters and prothrombin time. In contrast, aPTT was only normalised by aDabi-Fab. Plasma concentration (activity) of dabigatran remained elevated after PCC and rFVIla therapy, but was not measureable after aDabi-Fab.

Conclusion: In conclusion, PCC and aPCC were effective in reducing the anticoagulant effects of dabigatran under different conditions, while aDabi-Fab fully corrected all coagulation measures and decreased the plasma concentration of dabigatran below the limit of detection. No significant effects were observed with rFVlla.

\section{Introduction}

Uncontrolled post-traumatic bleeding is the leading cause of potentially preventable death among trauma patients [1]. Early coagulation abnormalities are frequent in severely injured trauma patients and are associated with substantially increased mortality [2]. Patients' tendency towards perioperative and trauma-induced bleeding is further increased by anticoagulant medication. The direct oral anticoagulant (DOAC) dabigatran, a direct thrombin inhibitor, is administered orally as the prodrug dabigatran etexilate (DE) and is characterised by a

\footnotetext{
* Correspondence: ogrottke@ukaachen.de

'Department of Anaesthesiology, RWTH Aachen University Hospital,

Pauwelsstrasse 30, D-52074 Aachen, Germany

Full list of author information is available at the end of the article
}

rapid onset of action, few drug or food interactions, no requirement for routine coagulation monitoring and a short half-life of 12 to 14 hours [3].

Dabigatran is approved in many countries for the primary prevention of venous thromboembolic events in adult patients who have undergone elective total hip or total knee replacement surgery, and/or for the prevention of stroke and systemic embolism in adult patients with non-valvular atrial fibrillation [3,4]. Dabigatran directly inhibits both free and clot-bound thrombin, and this impedes the conversion of fibrinogen to fibrin, thus preventing thrombus development. Results from a large multicentre randomised controlled trial including 18,113 patients with atrial fibrillation (RE-LY) suggest a low overall risk of bleeding complications [5]. Because of this 
and the fact that clinical use of DOACs began relatively recently, experience in the management of bleeding complications associated with DOACs is limited. However, in the case of life-threatening bleeding following injury, immediate and prompt reversal of anticoagulation is required [6]. At the present time, no specific antidotes are licensed for reversal of the anticoagulant effects of dabigatran.

Experimental data show that dabigatran may be removed from the circulation by dialysis [7]. This approach is reported to be effective in patients with endstage renal disease and several case reports have been published showing that dialysis is effective in reducing plasma concentrations of dabigatran [8-10]. However, this procedure may not be feasible in haemodynamically unstable patients with haemorrhagic shock. Prothrombin complex concentrate (PCC), activated PCC (aPCC) and recombinant activated factor VII (rFVIIa) have been proposed as candidates for reversing the anticoagulant effects of dabigatran. However, results from initial experimental trials are inconclusive and they do not account for different dabigatran concentrations or the combined effects of dabigatran and severe injury such as trauma [11-13]. Data from the few studies that have been performed in humans are also inconclusive. One study of PCC in healthy volunteers previously receiving dabigatran showed an increase in endogenous thrombin potential [14], whereas in another study PCC did not reverse the effect of dabigatran as measured by activated partial thromboplastin time (aPTT) [15]. A specific antibody fragment to dabigatran (aDabi-Fab) is in development, and in a rat model of anticoagulation it rapidly reversed the anticoagulant activity of dabigatran [16]. However, this antidote is not yet licensed for clinical use.

This porcine study was performed to evaluate the potential use of commonly available haemostatic agents (PCC, aPCC and rFVIIa), as well as the specific antidote aDabi-Fab, to reverse dabigatran-induced coagulopathy in an anticoagulation/trauma model. In addition, the study investigated the sensitivity of different coagulation tests, including thromboelastometry variables, for diagnosis and reversal of dabigatran/trauma-induced coagulopathy.

\section{Materials and methods}

\section{Ethics and anaesthesia}

All experiments were performed in accordance with German legislation governing animal studies following the Principles of Laboratory Animal Care. Ethical approval for these studies was obtained from the regional governmental animal care and use office (No. 84-02.04.2012.A197). Before surgery, pigs were housed in ventilated rooms and allowed to acclimatise to their surroundings for a minimum of seven days. Animals were fasted overnight before surgical procedures, with unrestricted access to water.
Prior to surgery, DE (Pradaxa, Boehringer Ingelheim, Biberach, Germany) was administered orally twice daily for 3 days $(30 \mathrm{mg} / \mathrm{kg} \mathrm{bid})$. On the day of surgery, animals received an intramuscular injection of $4 \mathrm{mg} / \mathrm{kg}$ azaperone (Stresnil, Janssen, Neuss, Germany) and $0.1 \mathrm{mg} / \mathrm{kg}$ atropine (atropine sulphate, B Braun, Melsungen, Germany) as premedication. Anaesthesia was induced by intravenous injection of $3 \mathrm{mg} / \mathrm{kg}$ propofol (Disoprivan, Astra Zeneca, Wedel, Germany) followed by orotracheal intubation. The animals were ventilated with a tidal volume of $8 \mathrm{~mL} / \mathrm{kg}$ and 16 to 22 breaths/minute (Cato, Draeger, Luebeck, Germany) to maintain end-tidal carbon dioxide between 36 and $42 \mathrm{mmHg}$. Anaesthesia was maintained with isoflurane (Forane, Abbott Laboratories Inc., Abbott Park, IL, USA) at an end-tidal concentration of $1 \%$ and a continuous infusion of fentanyl (Janssen, Neuss, Germany) at 3 to $4 \mu \mathrm{g} / \mathrm{kg} / \mathrm{h}$. Ringer's solution (Sterofundin, Braun, Germany) was infused at $4 \mathrm{~mL} / \mathrm{kg} / \mathrm{h}$ initially, increasing to $10 \mathrm{~mL} / \mathrm{kg} / \mathrm{h}$ after laparotomy until infliction of trauma. Throughout the experiment, body temperature was maintained at 36.5 to $37.0^{\circ} \mathrm{C}$ with a warming blanket.

Monitoring included electrocardiography (ECG), tail pulse oximetry, temperature, and arterial and central venous pressure measured by femoral catheters connected to a standard anaesthesia monitor (AS/3, Datex Ohmeda, Helsinki, Finland).

\section{Surgical preparation and dabigatran infusion}

Two 8.5-Fr catheters were surgically implanted in the right and left jugular veins for volume substitution and insertion of a pulmonary artery catheter. The right femoral artery was cannulated with an 18-G catheter to collect blood samples and to measure continuous arterial pressure. After line placement, a midline laparotomy with cystostomy was performed. Subsequently, dabigatran (active substance; Boehringer Ingelheim, Biberach, Germany) was infused at a rate of $0.77 \mathrm{mg} / \mathrm{kg} / \mathrm{h}$ for 30 minutes and $0.52 \mathrm{mg} / \mathrm{kg} / \mathrm{h}$ for 60 minutes to achieve consistent, supratherapeutic plasma concentrations of dabigatran.

A reproducible blunt liver injury was induced by clamping once through the parenchyma of the right middle liver lobe, using a custom-made instrument; the procedure has been described previously by our group [17]. Five minutes after injury and following haemorrhagic shock, all animals received a fluid bolus of $35 \mathrm{~mL} / \mathrm{kg}$ of Ringer's solution followed by continuous infusion of $40 \mathrm{~mL} / \mathrm{kg} / \mathrm{h}$ until four hours post-injury. Sixty minutes after trauma, the abdomen was reopened and the blood was collected to determine the total blood loss post-injury.

\section{Haemostatic agents}

The following haemostatic agents were tested: PCC (Beriplex P/N 250 (US brand-name Kcentra), CSL Behring GmbH, 
Marburg, Germany; lot 56560111C), aPCC (FEIBA, Baxter, Vienna, Austria; lot VNP5L003), and rFVIIa (NovoSeven, NovoNordisk, Denmark; lot LR04350). In addition, a specific antibody fragment to dabigatran being developed as an antidote (aDabi-Fab, Boehringer Ingelheim, Biberach, Germany; lot 6001325) was also tested [16]. PCC, aPCC and rFVIIa were reconstituted with sterile water according to the manufacturer's instructions immediately prior to administration. The aDabi-Fab was obtained in Tween 20 buffer $(25 \mathrm{mM}$ acetate, $220 \mathrm{mM}$ sorbitol and $0.2 \%$ polysorbate 20 ) at a concentration of $44 \mathrm{mg} / \mathrm{mL}$. Aliquots were stored at $-80^{\circ} \mathrm{C}$ and thawed at $37^{\circ} \mathrm{C}$ for 10 minutes prior to application.

\section{Blood collection and ex vivo addition of haemostatic agents}

Blood samples were collected into sodium citrate (Sarsted, Nuembrecht, Germany) at the following four time points: baseline (3 days before oral administration of dabigatran was started), $12 \mathrm{~h}$ after the last oral dose of DE, which represents trough levels of dabigatran (low dabigatran level), after the 90-minute dabigatran infusion, which represents peak levels of dabigatran (high dabigatran level) and 60 minutes post-injury (post-trauma), which was also 60 minutes after stopping the dabigatran infusion and induction of blunt trauma injury. Placebo (saline), PCC, aPCC, rFVIIa or aDabi-Fab was added ex vivo to each citrated whole blood sample from each time point. The concentration of PCC and aPCC added was equivalent to the plasma concentrations achieved with $30 \mathrm{U} / \mathrm{kg}$ and $60 \mathrm{U} / \mathrm{kg}$; rFVIIa was similarly added to achieve plasma levels equivalent to those achieved with $90 \mu \mathrm{g} / \mathrm{kg}$ and $180 \mu \mathrm{g} / \mathrm{kg}$. aDabi-Fab was added at a concentration to achieve plasma levels equivalent to 30 or $60 \mathrm{mg} / \mathrm{kg}$.

\section{Analytical methods including coagulation assays and thromboelastometry}

Haemoglobin $(\mathrm{Hb})$ concentrations were measured with a blood gas analyser (ABL500, Radiometer, Copenhagen, Denmark). Prothrombin time (PT, Innovin), aPTT (Actin FS) and fibrinogen concentration (thrombin reagent) were determined by standard laboratory methods using the appropriate tests (all from Dade Behring, Marburg, Germany) on a coagulometer (MC 4 plus, Merlin Medical, Lemgo, Germany). Dabigatran plasma concentration was determined using the diluted thrombin time (Hemoclot, HyphenBiomed, Neuville sur-Oise, France).

Coagulation was assessed in whole blood using a thromboelastometry device (ROTEM, Tem International $\mathrm{GmbH}$, Munich, Germany) and the EXTEM assay. The following parameters were measured: clotting time (CT, s), clot formation time (CFT, s) and maximum clot firmness (MCF, mm).

\section{Statistical analysis}

Statistical analysis was performed using PASW 18 (SPSS, Chicago, IL, USA). For graphical purposes, GraphPad Prism (Version 6.0, GraphPad Software, Inc., La Jolla, CA, USA) was used. Differences between the control and intervention groups were analysed with a one-way analysis of variance (ANOVA), with the Dunnett post hoc test for multiple comparisons. 'Non-measurable' was entered for clot formation time (CFT) when the required clot amplitude of $20 \mathrm{~mm}$ was not reached within 4,000 seconds. Data are presented as mean \pm SD. Statistical tests were performed two-tailed and $P$-values $<0.05$ were considered statistically significant.

\section{Results}

Five male German land-race pigs were included in this ex vivo study; the animals' bodyweights ranged between 37 and $42 \mathrm{~kg}$.

\section{Effects of oral administration of DE and intravenous infusion of dabigatran}

All coagulation parameters were within reference ranges at baseline (grey dotted line in all figures). After three days of oral DE, the mean plasma concentration of dabigatran was $380 \pm 106 \mathrm{ng} / \mathrm{mL}$ (low dabigatran, in Table 1). Laboratory coagulation parameters were prolonged compared with baseline: PT from $9 \pm 1$ to $25 \pm 8 \mathrm{~s}$ and aPTT from $13 \pm 1$ to $22 \pm 4 \mathrm{~s}$ (control, Figures $1 \mathrm{~A}$ and $2 \mathrm{~A}$ ). Accordingly, the EXTEM variables CT and CFT were also substantially prolonged (control, Figure 3A and B). However, no effects of oral DE administration on clot strength (MCF) or concentration of haemoglobin, platelets or fibrinogen were observed (control, Figure $3 \mathrm{C}$ and Table 2).

Following the 90-minute infusion of dabigatran, the mean plasma concentration (activity) of dabigatran increased to $1423 \pm 432 \mathrm{ng} / \mathrm{mL}$. This supratherapeutic level was associated with a further prolongation of PT, aPTT, and the EXTEM variables $\mathrm{CT}$ and CFT (Figures $1 \mathrm{~B}, 2 \mathrm{~B}$ and 4 ). These changes in coagulation parameters were compounded by blood loss following trauma (total blood loss at 60 minutes $1978 \pm 265 \mathrm{~mL}$ ) and dilution following the infusion of crystalloids. Sixty minutes after trauma, four out of five animals had no measurable clot formation (EXTEM CFT $\geq 4,000 \mathrm{~s}$ ), and clot strength (EXTEM MCF) had reduced to $11 \pm 7 \mathrm{~mm}$ (Figure 5). At the same time, plasma fibrinogen concentration had decreased to $64 \pm$ $12 \mathrm{mg} / \mathrm{dL}$ and the mean haemoglobin level had dropped to $4.5 \pm 0.6 \mathrm{~g} / \mathrm{L}$ (Table 2). In addition, further prolongation of $\mathrm{PT}$ and aPTT was seen (control, Figures $1 \mathrm{C}$ and $2 \mathrm{C}$ ).

\section{Measurements after haemostatic therapy ex vivo Ex-vivo treatment with $P C C$}

The effects of dabigatran anticoagulation, alone (after both oral and intravenous administration) and compounded by 
Table 1 Plasma concentration (activity, measured by diluted thrombin time) of dabigatran $(\mathrm{ng} / \mathrm{mL}$ ) during the study

\begin{tabular}{llll}
\hline & Low dabigatran & High dabigatran & Post-trauma \\
\hline Control & $380 \pm 106$ & $1423 \pm 432$ & $1021 \pm 238$ \\
PCC $(30 ; 60 \mathrm{U} / \mathrm{kg})$ & $299 \pm 101 ; 302 \pm 115$ & $1276 \pm 443 ; 1273 \pm 479$ & $814 \pm 81 ; 801 \pm 185$ \\
aPCC $(30 ; 60 \mathrm{U} / \mathrm{kg})$ & $291 \pm 108 ; 288 \pm 107$ & $1199 \pm 452 ; 1188 \pm 449$ & $785 \pm 166 ; 795 \pm 159$ \\
rFVIla $(90 ; 180 \mathrm{\mu g} / \mathrm{kg})$ & $270 \pm 86 ; 267 \pm 87$ & $1266 \pm 500 ; 1295 \pm 496$ & $766 \pm 194 ; 797 \pm 161$ \\
aDabi-Fab $(30 ; 60 \mathrm{mg} / \mathrm{kg})$ & $0.0 \pm 0.0 ; 0.0 \pm 0.0$ & $0.0 \pm 0.0 ; 0.0 \pm 0.0$ & $0.0 \pm 0.0 ; 0.0 \pm 0.0$ \\
\hline
\end{tabular}

Because dabigatran etexilate had not been administered, plasma concentrations of dabigatran were not measured at baseline.

Data are shown as mean \pm SD. PCC, prothrombin complex concentrate; aPCC, activated PCC; rFVIla, recombinant activated factor VII; aDabi-Fab, antibody fragment to dabigatran.

trauma-induced coagulopathy, were reduced by PCC, as shown by significant decreases versus control in PT, CT and CFT (Figures 1, 3, 4 and 5). Increased effects were apparent with the higher concentration of PCC after intravenous administration of dabigatran (high plasma concentration of dabigatran) and, in addition to the effects on PT, CT and CFT, clot strength (EXTEM MCF) almost returned to baseline with both doses of PCC. A similar pattern was observed following trauma: PT, CT and CFT were significantly shortened by PCC, and clot strength was returned to levels close to baseline (Figures $1 \mathrm{C}$ and $5 \mathrm{~A}-\mathrm{C}$ ). Despite approximately 80 to $90 \%$ reversal of the effects on these parameters, $\mathrm{PCC}$ treatment had no effect on aPTT at any time point (Figure $2 \mathrm{~A}-\mathrm{C}$ ), and the plasma level (activity, measured by diluted thrombin time) of dabigatran was also unchanged by PCC.

\section{Ex vivo treatment with aPCC}

Dabigatran-induced coagulopathy, both after oral intake and infusion, was reversed by aPCC application, with very similar response patterns to those observed with PCC (Figures 1, 3, 4 and 5). Decreases in PT, CT and CFT were observed, and MCF returned close to baseline after intravenous dabigatran and trauma, although, as with PCC these parameters were not fully restored to baseline levels. Also as with PCC, aPCC had no significant effect on aPTT or levels of dabigatran.

\section{Ex vivo treatment with $r F V I I a$}

$E x$-vivo addition of rFVIIa had no significant effect on any of the coagulation parameters at any time point. This was true for both doses of rFVIIa. In addition, rFVIIa had no impact on the plasma concentration of dabigatran at any time point.

\section{Ex vivo treatment with aDabi-Fab}

After oral DE and intravenous dabigatran, both concentrations of aDabi-Fab restored coagulation parameters to their baseline values or similar. Even after trauma, PT, aPTT, CT, and CFT were reversed to baseline values (Figures $1 \mathrm{C}, 2 \mathrm{C}$ and 5), although a small decrease in MCF was still apparent. The restoration of aPTT to baseline values (Figure 2A-C) distinguished aDabi-Fab from PCC and aPCC. In addition, the mean plasma concentration of dabigatran was below the limit of detection (that is, approximately zero) after the addition of both concentrations of aDabi-Fab (Table 1).

\section{Discussion}

This experimental animal trial is the first to show in vitro that PCC, aPCC and aDabi-Fab are effective for the reversal of dabigatran-induced coagulopathy in a model of anticoagulation and liver trauma. Coagulation parameters, which were all affected by dabigatran administration, were corrected most effectively by aDabiFab, but, this product is yet to be licensed for clinical use. It is therefore of potential clinical importance that this study also suggests that PCC and aPCC could potentially be used for emergency reversal of dabigatran. In contrast, rFVIIa had no significant impact on coagulation after oral DE or intravenous dabigatran administration, despite the use of relatively high-dose rFVIIa (equivalent to $180 \mu \mathrm{g} / \mathrm{kg}$ ).

Theoretically, the use of prothrombin (a major component of PCC) may reverse the anticoagulation activity of dabigatran by providing thrombin for the transformation of fibrinogen to fibrin. PCCs and aPCCs are derived from human plasma and most contain the vitamin K-dependent coagulation factors II, VII, IX, and X. PCCs with low levels of factor VII (3-factor PCCs) are commonly used in the USA, but products with higher levels of factor VII (4-factor PCCs) are used more commonly outside the USA. Based on the results of an open-label, phase IIIb randomised controlled trial [18], the first 4-factor PCC (Kcentra, CSL Behring, Germany) has been recently licensed in the US for the urgent reversal of coagulation factor deficiency induced by vitamin $\mathrm{K}$ antagonist (for example, warfarin) therapy in adult patients with acute major bleeding $[19,20]$. Currently, there are two major indications for PCC: rapid reversal of oral anticoagulation (vitamin $\mathrm{K}$ antagonists) and deficiency of vitamin K-dependent coagulation factors in life-threatening bleeding. Unlike 4-factor or 3-factor PCCs, aPCC (FEIBA, Baxter, USA) contains activated factors and is indicated for haemophilia A and haemophilia B patients with inhibitors, either to control spontaneous 


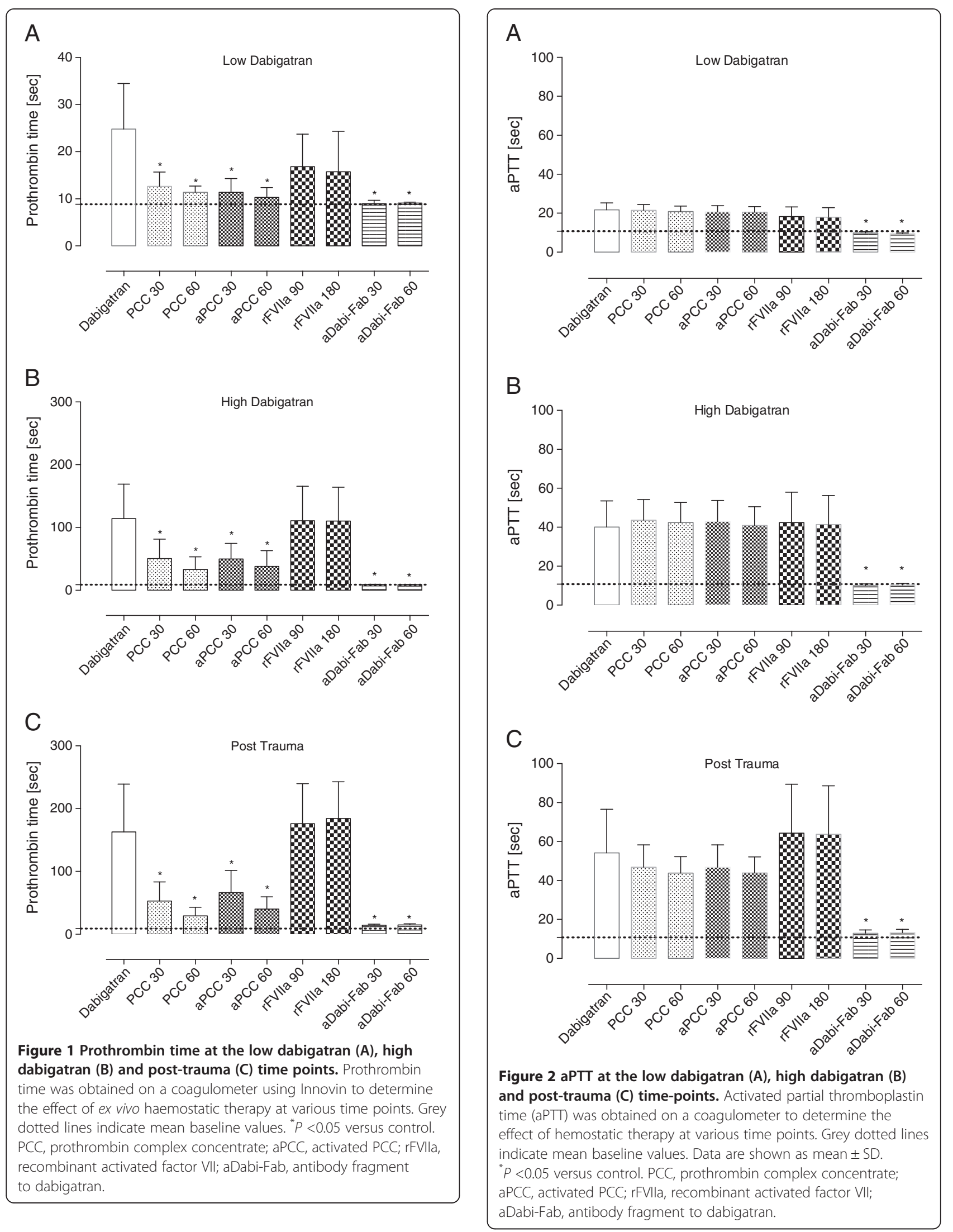




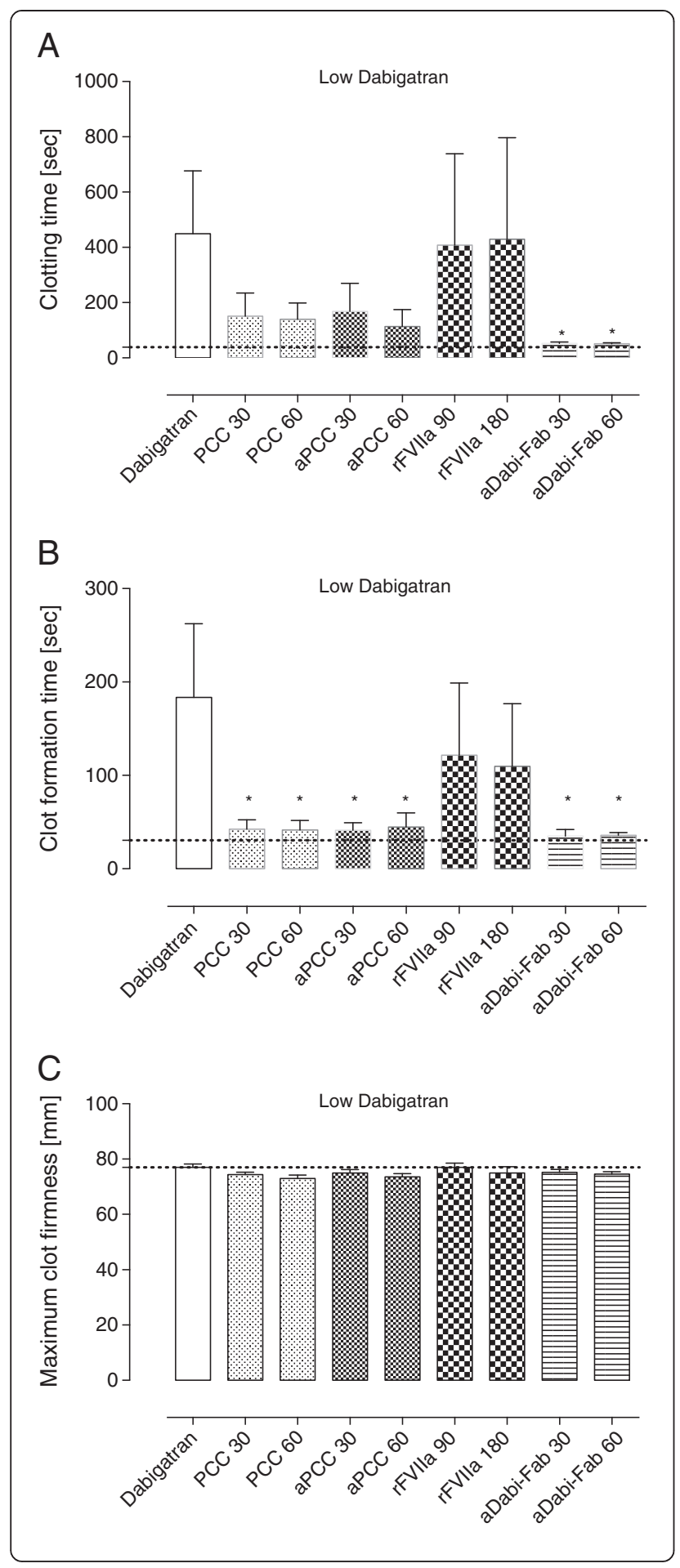

Figure $\mathbf{3}$ Thromboelastometry parameters after oral administration of dabigatran etexilate. The ROTEM coagulation analyzer (TEM international, Munich, Germany) was used for thromboelastometry. For activation the ExTEM reagent containing tissue factor as starting reagent was used according to the manufacturer instructions. (A) The clotting time (CT) represents the initiation of clot formation and corresponds to the reaction time. (B) clot formation time (CFT) reflects the coagulation time until $20 \mathrm{~mm}$ of amplitude are reached. (C) The maximum clot firmness (MCF) reflects the strength of a resulting clot. Data are shown as mean \pm SD. ${ }^{*} P<0.05$ versus control. PCC, prothrombin complex concentrate; aPCC, activated PCC; rFVIla, recombinant activated factor VIl; aDabi-Fab, antibody fragment to dabigatran.

bleeding episodes or for use during surgical interventions [21].

PCC has been successfully used to terminate serious bleeding in pre-clinical studies [22-24]. Evidence from pre-clinical studies also supports a potential role for the use of PCC to reverse the effects of dabigatran [11-13]. In a rabbit model of anticoagulation associated with dabigatran, the use of PCC significantly reduced blood loss in a dose-dependent manner following a standardised kidney incision [11]. Furthermore, in a murine model of intracerebral haemorrhage, animals receiving dabigatran twice daily showed an expansion of intracerebral haematoma on magnetic resonance imaging [13]. The use of PCC (up to $100 \mathrm{U} / \mathrm{kg}$ ) was associated with dose-dependent prevention of haematoma growth and also reversal of prolonged tail bleeding time. In another murine study, administration of PCC in combination with rFVIIa or aPCC prior to tail tip resection significantly reduced bleeding time but had no significant impact on blood loss, although the dose of PCC used in this study was low (14.3 U/kg) [12].

Few data exist on the use of PCC for the reversal of the anticoagulant effects of dabigatran in humans. In one study, following oral administration of dabigatran to 10 healthy volunteers, the capacity of thrombin generation was evaluated ex vivo. The intake of dabigatran was shown to affect the kinetics of thrombin generation, with a prolongation of the lag time and time to peak [14]. The addition of PCC significantly increased the endogenous thrombin potential, although no influence on the lag time was observed. In contrast to this study, in a trial of six healthy volunteers, PCC did not reverse the prolongation of the aPTT resulting from standard oral DE doses of $150 \mathrm{mg}$ twice daily [15].

Although the investigators in the latter study used a different PCC (Cofact; Sanquin Blood Supply, Amsterdam, the Netherlands) to the PCC used in this study (Beriplex P/N 250, CSL Behring GmbH, Marburg, Germany), we have previously shown the two PCCs to have a similar pattern of thrombin generation [25]. Thus, discrepancy between studies regarding the effect of PCC on plasma 
Table 2 Haematological parameters and fibrinogen concentration during the study

\begin{tabular}{lllll}
\hline & Baseline & Low dabigatran & High dabigatran & Post-trauma \\
\hline Haemoglobin $(\mathrm{g} / \mathrm{L})$ & $10.5 \pm 0.7$ & $10.2 \pm 0.8$ & $9.5 \pm 0.4$ & $4.5 \pm 0.7$ \\
Platelets $\left(\times 10^{3} / \mathrm{LL}\right)$ & $388 \pm 51$ & $403 \pm 36$ & $355 \pm 66$ & $202 \pm 50$ \\
Fibrinogen $(\mathrm{mg} / \mathrm{dL})$ & $161 \pm 16$ & $135 \pm 13$ & $118 \pm 19$ & $64 \pm 12$ \\
\hline
\end{tabular}

Data are shown as mean \pm SD.

coagulation assays raises the question of which coagulation test is adequate for monitoring the potential of a PCC to reverse the anticoagulant effects of dabigatran. The reversal of the prolonged PT, but not prolonged aPTT by $\mathrm{PCC} / \mathrm{aPCC}$ in this study highlights the need for an assay that is sensitive not only to the anticoagulant used, but also to the effects of the reversal agent. In the case of warfarin, the PT (or international normalized ratio) is dependent on the vitamin K-dependent coagulation factors [26] and therefore serves not only as a sensitive measure of the effects of anticoagulation therapy, but also of its reversal by PCCs or aPCC. Conversely, PT is an insensitive measure of the effects of therapeutic doses of dabigatran [26-28]. However, as shown in this study, a high concentration of the drug can prolong the PT, thus enabling the dabigatran-prolonged $\mathrm{PT}$ to be reversed by PCC and aPCC. aPTT is more sensitive than PT to therapeutic levels of dabigatran $[26,28]$, but the lack of effects of PCCs and aPCCs on aPTT suggests that this test is not suitable for monitoring reversal with these products. For confirmation of reversal of dabigatran therapy in the presence of trauma-related bleeding, well-designed studies with appropriate (sensitive) coagulation assays are urgently warranted.

In this study, we used thromboelastometry to monitor the effects of dabigatran. In the trauma setting, delays in the detection of coagulopathy may influence outcome; in contrast to the conventional coagulation tests, which are associated with slower turnaround times, thromboelastometry allows rapid assessment of a patient's coagulopathy. Based on findings from retrospective studies, thromboelastometry appears to be a useful tool to guide PCC therapy in patients with traumatic coagulopathy $[29,30]$. The EXTEM assay is similar to PT in that it assesses tissue factor-initiated extrinsic coagulation, making it the most suitable thromboelastometric assay for investigating PCC or aPCC reversal of dabigatran. There has been little investigation of the effects of dabigatran on viscoelastic coagulation parameters but, consistent with the present findings, prolonged activated clotting time with rapid thromboelastography was seen in several patients taking dabigatran [31]. We found that PCC, aPCC and aDabiFab reversed the anticoagulant effects of dabigatran as shown by improvements in CT and CFT. It is important to note that the decrease in MCF observed after dabigatran infusion was mainly attributable to a decrease in the amount of thrombin available for the conversion of fibrinogen to fibrin, as opposed to insufficient clot substrate (fibrinogen, platelets). Once sufficient thrombin becomes available through addition of PCC, aPCC or aDabi-Fab, transformation of fibrinogen to fibrin is restored and clot formation is no longer impaired. Overall, thromboelastometry may be useful in the detection of coagulopathy associated with dabigatran and in monitoring the effects of reversal therapy.

A drawback of PCC use is the potential risk of thromboembolic complications [24]. We have shown in an experimental animal model of liver injury that high levels $(50 \mathrm{U} / \mathrm{kg})$ of PCC may increase the risk for disseminated intravascular coagulation, and this was explained by an imbalance of pro- and anti-coagulant proteins [24]. However, there has been a suggestion that the procoagulant effects of highdose PCC are reduced in the presence of dabigatran [32]; the safety profile of PCC or aPCC for the reversal of dabigatran remains to be characterised.

rFVIIa is approved to treat haemophilia patients with inhibiting antibodies against coagulation factors VIII or IX [33]. The use of this agent in patients with bleeding trauma who are resistant to conventional haemostatic therapy, even as a last resort, may be considered as surprising given that a large randomised controlled trial (CONTROL) was stopped after an analysis predicted that the likelihood of a successful outcome concerning the primary endpoints (mortality and morbidity) was very low [34]. In the present study, rFVIIa had no effect on reversal of any coagulation parameters. This is consistent with previous data demonstrating lack of effect of rFVIIa on dabigatran-induced bleeding in a murine model of haemorrhagic stroke [13]. These findings may be explained by the mechanism of action of rFVIIa: it influences the kinetics of thrombin generation, but it does not increase the concentration of prothrombin.

The results of this in vitro study have shown the specific antidote to dabigatran, aDabi-Fab, to have the greatest impact on all coagulation variables at all measured time points. Dabigatran, even at supratherapeutic concentrations $(1,400$ to $1,500 \mathrm{ng} / \mathrm{mL}$, compared with the normal therapeutic range in humans of 50 to $250 \mathrm{ng} / \mathrm{mL}$ ), was completely neutralised after the addition of aDabi-Fab. This is in line with results from a previous rat model of anticoagulation in which a single bolus injection of aDabiFab completely reversed the anticoagulant activity of 


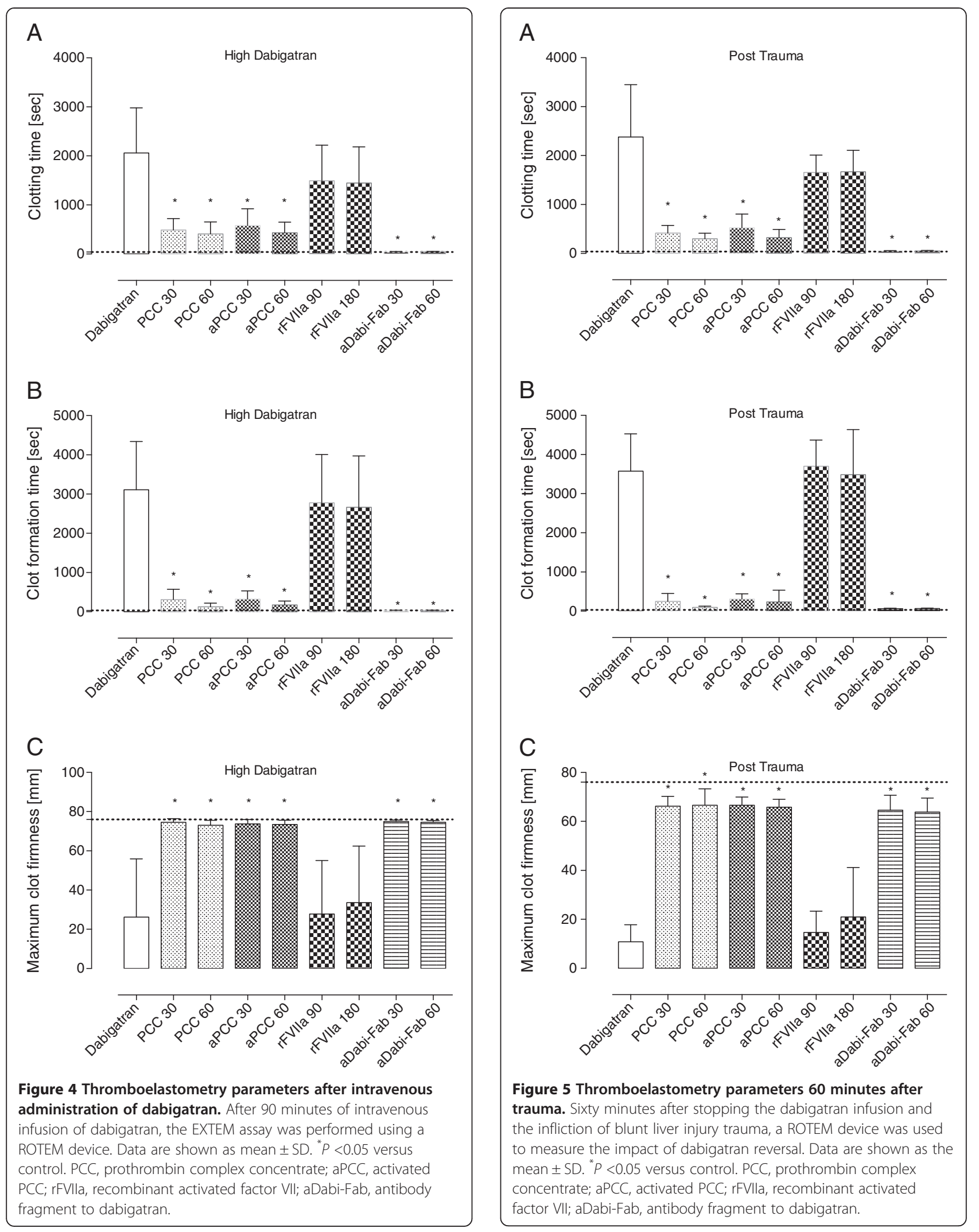


dabigatran. This reversal was maintained for approximately 25 minutes despite continued infusion of dabigatran [16]. The increased potency of aDabi-Fab in comparison with PCC, aPCC and rFVIIa is undoubtedly related to their different mechanisms of action. The aDabi-Fab binds directly to dabigatran in plasma or whole blood and inactivates it, thus all coagulation measurements are returned to baseline. In contrast, PCC, aPCC and rFVIIa do not bind to dabigatran. Instead, these drugs overcome the anticoagulant activity by increasing the availability of substrate for coagulation.

There are some limitations of our study that need to be acknowledged. Haemostatic agents and aDabi-Fab were supplemented ex vivo. This approach was taken mainly for ethical reasons: it enabled investigation of a variety of haemostatic agents with a small number of animals. As a consequence, however, the effects of treatment on blood loss could not be measured and it is uncertain to what extent our observations may be predictive of clinical dabigatran reversal. This uncertainty was in part mitigated by the use of intravenous dabigatran in its active form rather than the orally administered prodrug DE. Thus, possible species-related differences in drug absorption and conversion to active form cannot have influenced the present data. However, there remains a possibility of species differences in aspects such as the tissue factor/rFVIIa complex, and the aPTT/PT responses to dabigatran [35]. Another consideration regarding applicability to the clinical setting is the plasma level of dabigatran. Mean levels at the low dabigatran timepoint were slightly above the normal therapeutic range, with higher values with high dabigatran and post-trauma. Although our study would have been improved by matching more closely the normal therapeutic range of dabigatran levels, it seems reasonable to assume that if reversal is successful at supratherapeutic concentrations, it would also be successful at lower, therapeutic levels. Clinical data from human patients receiving dabigatran therapy are clearly needed to confirm the efficacy, optimal dosing and safety of PCC, aPCC and aDabi-Fab for the reversal of dabigatran.

\section{Conclusion}

In summary, dabigatran-induced coagulopathy was reversed by ex vivo administration of two different doses of exogenous PCC and aPCC in this porcine model of anticoagulation and blunt liver injury. In contrast, ex vivo rFVIIa had no significant effect on coagulation parameters after administration of dabigatran. Unlike PCC or aPCC, ex vivo addition of aDabi-Fab provided complete neutralisation of dabigatran as well as full reversal of its effects. However, aDabi-Fab is still in development and is therefore not yet available for clinical use. Although further data investigating the effects of aDabi-Fab and PCCs are urgently needed, the administration of PCC or aPCC could potentially be considered as a therapeutic option to control life-threatening bleeding among patients under treatment with dabigatran.

\section{Key messages}

- PCC and aPCC effectively reverse dabigatraninduced coagulopathy in an anticoagulation/liver trauma model.

- rFVIIa has no significant effect on coagulation in this setting.

- $\mathrm{aDabi}-\mathrm{Fab}$ provides the most effective reversal of the anticoagulant effects of dabigatran.

- Thromboelastometry variables may help to guide therapy in patients receiving dabigatran.

- Until aDabi-Fab becomes available, administration of PCC or aPCC might be a reasonable intervention for dabigatran-treated patients with life-threatening bleeding.

\section{Abbreviations}

aDabi-Fab: antibody fragment to dabigatran; ANOVA: analysis of variance; aPCC: activated prothrombin complex concentrate; aPTT: activated partial thromboplastin time; CFT: clot formation time; CT: clotting time;

DE: dabigatran etexilate; DOAC: direct oral anticoagulant;

ECG: electrocardiography; Hb: haemoglobin; MCF: maximum clot firmness;

PCC: prothrombin complex concentrate; PT: prothrombin time;

rFVIla: recombinant activated factor VII.

\section{Competing interests}

OG has received research funding from Novo Nordisk, Biotest, CSL Behring, Nycomed. He has also received honoraria for consultancy and/or travel support from Bayer Healthcare, Boehringer Ingelheim and CSL Behring. RR has received honoraria for lectures and consultancy from CSL Behring and Novo Nordisk. JvR is an employee of Boehringer Ingelheim Pharma GmbH \& Co., Germany. HS has received research funding from Boehringer Ingelheim and honoraria for consultancy from Bayer.

\section{Authors' contributions}

OG conceived and conducted the experimental laboratory work and drafted the manuscript. RR, HS and JVR helped to draft the manuscript. All authors read and approved the final manuscript.

\section{Acknowledgements}

The authors thank Renate Nadenau and Christian Beckers (both Department of Anaesthesiology, RWTH Aachen University Hospital) for excellent laboratory assistance.

This study was performed at the RWTH Aachen University Hospital, Pauwelsstrasse 30, D-52074 Aachen, Germany.

\section{Author details}

'Department of Anaesthesiology, RWTH Aachen University Hospital, Pauwelsstrasse 30, D-52074 Aachen, Germany. ${ }^{2}$ CardioMetabolic Diseases Research, Boehringer Ingelheim GmbH \& Co. KG, Birkendorfer Str 65, D-88397 Biberach, Germany. ${ }^{3}$ Laboratory for Clinical Thrombosis and Haemostasis, Department of Internal Medicine, Cardiovascular Research Institute Maastricht, Maastricht University Medical Center, 6200 MD Maastricht, The Netherlands.

Received: 21 October 2013 Accepted: 30 January 2014

Published: 5 February 2014

\section{References}

1. Holcomb JB: Optimal use of blood products in severely injured trauma patients. Hematology Am Soc Hematol Educ Program 2010, 2010:465-469. 
2. Maegele M, Lefering R, Yucel N, Tjardes T, Rixen D, Paffrath T, Simanski C, Neugebauer E, Bouillon B: Early coagulopathy in multiple injury: an analysis from the German Trauma Registry on 8724 patients. Injury 2007 38:298-304.

3. van Ryn J, Goss A, Hauel N, Wienen W, Priepke H, Nar H, Clemens A: The discovery of dabigatran etexilate. Frontiers in Pharmacology 2013, $4: 12$.

4. Dabigatran - Summary of product characteristics. [http://www.medicines. org.uk/emc/medicine/20760\#INDICATIONS] Accessed 18 October 2013.

5. Connolly SJ, Ezekowitz MD, Yusuf S, Eikelboom J, Oldgren J, Parekh A, Pogue J, Reilly PA, Themeles E, Varrone J, Wang S, Alings M, Xavier D, Zhu J, Diaz R, Lewis BS, Darius H, Diener HC, Joyner CD, Wallentin L, RE-LY Steering Committee and Investigators: Dabigatran versus warfarin in patients with atrial fibrillation. N Engl J Med 2009, 361:1139-1151.

6. Spahn DR, Bouillon B, Cerny V, Coats TJ, Duranteau J, Fernandez-Mondejar E, Filipescu D, Hunt BJ, Komadina R, Nardi G, Neugebauer E, Ozier Y, Riddez L, Schultz A, Vincent JL, Rossaint R: Management of bleeding and coagulopathy following major trauma: an updated European guideline. Crit Care 2013, 17:R76.

7. Liesenfeld KH, Staab A, Hartter S, Formella S, Clemens A, Lehr T: Pharmacometric characterization of dabigatran hemodialysis. Clin Pharmacokinet 2013, 52:453-462.

8. Khadzhynov D, Wagner F, Formella S, Wiegert E, Moschetti V, Slowinski T, Neumayer HH, Liesenfeld KH, Lehr T, Hartter S, Friedman J, Peters H, Clemens A: Effective elimination of dabigatran by haemodialysis, A phase I single-centre study in patients with end-stage renal disease. Thromb Haemost 2013, 109:596-605.

9. Esnault P, Gaillard PE, Cotte J, Cungi PJ, Beaume J, Prunet B: Haemodialysis before emergency surgery in a patient treated with dabigatran. $\mathrm{Br} J$ Anaesth 2013, 111:776-777.

10. Warkentin TE, Margetts P, Connolly SJ, Lamy A, Ricci C, Eikelboom JW: Recombinant factor Vlla (rFVlla) and hemodialysis to manage massive dabigatran-associated postcardiac surgery bleeding. Blood 2012, 119:2172-2174

11. Pragst I, Zeitler SH, Doerr B, Kaspereit FJ, Herzog E, Dickneite G, van Ryn J: Reversal of dabigatran anticoagulation by prothrombin complex concentrate (Beriplex P/N) in a rabbit model. J Thromb Haemost 2012, 10:1841-1848

12. Lambourne MD, Eltringham-Smith $\sqcup$, Gataiance $S$, Arnold DM, Crowther MA, Sheffield WP: Prothrombin complex concentrates reduce blood loss in murine coagulopathy induced by warfarin, but not in that induced by dabigatran etexilate. J Thromb Haemost 2012, 10:1830-1840.

13. Zhou W, Schwarting S, Illanes S, Liesz A, Middelhoff M, Zorn M, Bendszus M, Heiland S, van Ryn J, Veltkamp R: Hemostatic therapy in experimental intracerebral hemorrhage associated with the direct thrombin inhibitor dabigatran. Stroke 2011, 42:3594-3599.

14. Marlu R, Hodaj E, Paris A, Albaladejo P, Cracowski JL, Pernod G: Effect of non-specific reversal agents on anticoagulant activity of dabigatran and rivaroxaban: a randomised crossover ex vivo study in healthy volunteers. Thromb Haemost 2012, 108:217-224.

15. Eerenberg ES, Kamphuisen PW, Sijpkens MK, Meijers JC, Buller HR, Levi M: Reversal of rivaroxaban and dabigatran by prothrombin complex concentrate: a randomized, placebo-controlled, crossover study in healthy subjects. Circulation 2011, 124:1573-1579.

16. Schiele F, van Ryn J, Canada K, Newsome C, Sepulveda E, Park J, Nar H, Litzenburger T: A specific antidote for dabigatran: functional and structural characterization. Blood 2013, 121:3554-3562.

17. Grottke O, Braunschweig T, Philippen B, Gatzweiler KH, Gronloh N, Staat M, Rossaint R, Tolba R: A new model for blunt liver injuries in the swine. Eur Surg Res 2010, 44:65-73.

18. Sarode R, Milling TJ Jr, Refaai MA, Mangione A, Schneider A, Durn BL, Goldstein JN: Efficacy and Safety of a Four-Factor Prothrombin Complex Concentrate (4 F-PCC) in Patients on Vitamin K Antagonists Presenting with Major Bleeding: A Randomized, Plasma-Controlled, Phase Illb Study. Circulation 2013, 128:1234-1243.

19. Kcentra prescribing information. [http://labeling.cslbehring.com/PI/US/ Kcentra/EN/Kcentra-Prescribing-Information.pdf] Accessed 18 October 2013.

20. CSL Behring Receives FDA Approval of Kcentra ${ }^{\mathrm{TM}}$ for Urgent Warfarin Reversal in Patients with Acute Major Bleeding. [http://www.cslbehring. com/s1/cs/enco/1151517263302/news/1255931252507/prdetail.htm] Accessed 18 October 2013
21. FEIBA - Summary of product characteristics. [http://www.haemophiliacare. co.uk/pdfs/FElBA_summary_product.pdf] Accessed 18 October 2013.

22. Dickneite G, Pragst I: Prothrombin complex concentrate vs fresh frozen plasma for reversal of dilutional coagulopathy in a porcine trauma model. Br J Anaesth 2009, 102:345-354.

23. Honickel M, Rieg A, Rossaint R, Braunschweig $T$, Spronk HM, ten Cate $H$, van Oerle R, Tolba R, Grottke O: Prothrombin complex concentrate reduces blood loss and enhances thrombin generation in a pig model with blunt liver injury under severe hypothermia. Thromb Haemost 2011, 106:724-733.

24. Grottke O, Braunschweig T, Spronk HM, Esch S, Rieg AD, van Oerle R, ten Cate H, Fitzner C, Tolba R, Rossaint R: Increasing concentrations of prothrombin complex concentrate induce disseminated intravascular coagulation in a pig model of coagulopathy with blunt liver injury. Blood 2011, 118:1943-1951.

25. Grottke O, Rossaint R, Henskens $Y$, van Oerle R, Ten Cate H, Spronk HM: Thrombin generation capacity of prothrombin complex concentrate in an in vitro dilutional model. PloS One 2013, 8:e64100.

26. Favaloro EJ, Bonar R, Butler J, Marsden K: Laboratory testing for the new oral anticoagulants: a review of current practice. Pathology 2013, 45:435-437.

27. Eby C: Novel anticoagulants and laboratory testing. Int J Lab Hematol 2013, 35:262-268.

28. van Ryn J, Stangier J, Haertter S, Liesenfeld KH, Wienen W, Feuring M, Clemens A: Dabigatran etexilate-a novel, reversible, oral direct thrombin inhibitor: interpretation of coagulation assays and reversal of anticoagulant activity. Thromb Haemost 2010, 103:1116-1127.

29. Schochl H, Nienaber U, Maegele M, Hochleitner G, Primavesi F, Steitz B, Arndt C, Hanke A, Voelckel W, Solomon C: Transfusion in trauma: thromboelastometry-guided coagulation factor concentrate-based therapy versus standard fresh frozen plasma-based therapy. Crit Care 2011, 15:R83.

30. Schochl H, Forster L, Woidke R, Solomon C, Voelckel W: Use of rotation thromboelastometry (ROTEM) to achieve successful treatment of polytrauma with fibrinogen concentrate and prothrombin complex concentrate. Anaesthesia 2010, 65:199-203.

31. Cotton BA, McCarthy JJ, Holcomb JB: Acutely injured patients on dabigatran. N Engl J Med 2011, 365:2039-2040.

32. Herzog E, Kaspereit F, Krege W, van Ryn J, Dickneite G, Pragst I: Non-clinical safety and efficacy of prothrombin complex concentrates (PCC) for the reversal of dabigatran mediated anticoagulation. J Thromb Haemost 2013, 11:Abstr PB 2.48-3.

33. Hedner U: Treatment of patients with factor VIII and factor IX inhibitors with special focus on the use of recombinant factor Vlla. Thromb Haemost 1999, 82:531-539.

34. Hauser CJ, Boffard K, Dutton R, Bernard GR, Croce MA, Holcomb JB, Leppaniemi A, Parr M, Vincent JL, Tortella BJ, Dimsits J, Bouillon B, CONTROL Study Group: Results of the CONTROL trial: efficacy and safety of recombinant activated Factor VII in the management of refractory traumatic hemorrhage. J Trauma 2010, 69:489-500.

35. Tanaka KA, Bolliger D: On the reversal of new oral anti-coagulants: can we simply extrapolate data from the animal models to humans? $\mathrm{Br} J$ Anaesth 2013, 110:329-332.

doi:10.1186/cc13717

Cite this article as: Grottke et al:: Prothrombin complex concentrates and a specific antidote to dabigatran are effective ex-vivo in reversing the effects of dabigatran in an anticoagulation/liver trauma experimental model. Critical Care 2014 18:R27. 